\title{
ANALISIS CUALITATIVO DE LA SOLIDIFICACION DEL SISTEMA BINARIO PbSb
}

\author{
L. Flores ${ }^{\text {a }}$ y J. Gómez ${ }^{\text {a }}$ \\ ${ }^{a}$ Instituto de Investigación de Física, Universidad Nacional Mayor de San Marcos, Apartado postal 14-0149, \\ Av. Venezuela Cuadra 32, Lima, Perú.
}

\begin{abstract}
Resumen
Al enfriar una aleación $\mathrm{PbSb}$ desde la temperatura de colada, el tamaño de los granos estará determinado por la relación entre la rapidez de crecimiento y la rapidez de nucleación. Se utilizó las curvas de enfriamiento y se realizó un análisis metalográfico para identificar la microestructura de ésta, utilizando el balance de calor se calculó el calor latente de fusión. El estudio de la transferencia calórica en un sistema metal-molde se realizó mediante soluciones numéricas discretas, considerando las transformación de fase líquido-sólido y la convexión natural entre la fase líquida y pastosa de la aleación. Los métodos utilizados fueron el método de volumen finito y el método de línea a línea. Se observa un subenfriamiento en la muestra 3, debido a la lenta velocidad de enfriamiento, además el tamaño de grano es mayor en la muestra 1 y la solidificación se forma 2 fases sólidas, con una estructura eutéctica (formado por las fases $\alpha$ y $\beta$ ) y la fase $\beta$ primaria.
\end{abstract}

Palabras claves: solidificación, nucleación, método numérico, estructura eutéctica.

\begin{abstract}
When to chill an alloy PbSb since the temperature of strained, the size of the grains will be determined by the relation between the rapidity of growth and the rapidity of nucleation. It was utilized the curves of cooling off and an analysis was carried out metallographic to identify the microstructure of this, utilizing the balance of heat was calculated the latent heat of fusion. The study of the caloric transfer in a system metal-mold was carried out by means of discreet numerical solutions, considering the transformation of liquid-solid phase and the convexion natural between the phase liquid and thick of the alloy. The methods utilized were the method of finite volume and the method of line to line. A subcooling off in the sample is observed 3, due to the slow velocity of cooling off, also the size of grain is greater in the sample 1 and the solidification is formed 2 solid phases, with a structure eutectic (formed by the phases $\alpha$ and $\beta$ ) and the phase $\beta$ primary.
\end{abstract}

Keywords: Solidification, nucleation, numerical method, eutectic structure.

\section{Introducción.}

La solidificación se puede estudiar con diferentes técnicas experimentales, como análisis térmico y solidificación direccional. El análisis térmico se basa en el enfriamiento o calentamiento de la aleación que se estudia y esta relacionado con las transformaciones de fase de estas. El estudio se realiza mediante la curva de enfriamiento, obtenido por colada de la aleación $\mathrm{PbSb}$, obteniéndose la velocidad de enfriamiento, calor latente y además se puede predecir la microestructura de esta aleación. El análisis metalográfico nos dirá como es la 
microestructura, el tamaño de granos y si existe alguna relación con la estructura hipereutectica. Mediante el cálculo numérico se simuló la solidificación de la aleación $P b S b$ y se calculó la curva de enfriamiento [1]. En el presente trabajo estudiaremos como ocurre la solidificación y el producto de ésta a diferentes temperaturas de colada.

\section{Teoría}

El tamaño de los granos en una pieza colada esta determinado por la relación entre la rapidez de crecimiento y la rapidez de nucleación. La rapidez de enfriamiento es el factor más importante para determinar la rapidez de nucleación y por lo tanto, el tamaño de grano. El enfriamiento rápido (colada fría) dará como resultado la formación de un gran número de núcleos y que se obtenga un tamaño de grano fino, en tanto que en el enfriamiento lento (fundición en arena o molde caliente) solo se forman algunos núcleos y tendrán la oportunidad de crecer, consumiendo el líquido antes que se puedan formar mas núcleos [1].

\subsection{Diagrama de fase y energía libre}

En un sistema de 2 componentes, ocurre máximo 3 fases en equilibrio bajo condiciones muy limitada, no es difícil ver la razón para esto, pues las energías molares parciales de los componentes deben ser las mismas en cada una de las 3 fases. Cada curva de energía libre varia con la temperatura en una manera diferente a las otras fases y en general, habrá una temperatura donde sea posible trazar una simple recta tangencial a las tres curvas, esta temperatura se llama temperatura eutéctica, entonces de una simple fase líquida se puede convertir a 2 fases sólidas; cuando las 3 fases están en equilibrio, la composición de cada fase es fija [2].

El crecimiento del sólido ocurre cuando los átomos del líquido se unen al núcleo hasta que se acaba el líquido. Se espera que un material solidifique cuando el líquido se enfríe por debajo de la temperatura de solidificación del mismo. Cuando esto ocurre, la energía asociada con la estructura cristalina del sólido es progresivamente menor que la energía del líquido, haciendo cada vez más estable al sólido conforme la temperatura disminuya. Una vez que se ha formado el núcleo sólido, el crecimiento depende de la forma en que se extrae el calor del sistema sólido-líquido [3].

\subsection{Método Analítico}

Ecuación de la energía

Para describir la solidificación $[5,6]$ de la aleación se utilizó la ecuación de la energía total, la cual es

$$
\rho C_{p} \frac{\partial T}{\partial t}+\nabla \cdot(\rho \vec{v} T)=\nabla \cdot(k \nabla T)+Q
$$

donde:

$$
\begin{aligned}
& \rho: \text { densidad del metal } \\
& k: \text { conductividad del metal } \\
& v: \text { velocidad del fluido } \\
& T: \text { temperatura } \\
& t: \text { tiempo } \\
& Q: \text { sumidero o fuente de calor } \\
& C_{p}: \text { calor específico } \\
& \rho C_{p} \frac{\partial T}{\partial t}: \text { calor transiente } \\
& \nabla \cdot(\rho \vec{v} T): \text { calor por convexión } \\
& \nabla \cdot(k \nabla T): \text { calor por conducción }
\end{aligned}
$$

\section{Ley de Newton}

La Ley de Newton describe el calor transferido desde la superficie de un sólido en contacto con el medio que lo rodea:

$$
q_{i}=h A_{s}\left(T_{s}-T_{a m b}\right)
$$

$q_{i}:$ calor por convexión de aire

$h$ : coeficiente convectivo.

$A_{S}$ : área de la superficie.

$T_{S}$ : temperatura de la superficie.

$T_{\text {amb. }}$ : temperatura ambiente.

\section{$\underline{\text { Balance de calor }}$}

Utilizando las ecuaciones (1) y (2) se realiza el balance de calor de la aleación, dando la forma:

$$
\rho V C_{p} \frac{d T}{d t}=h A\left(T-T_{a m b}\right)-K A \frac{\partial T}{\partial x} .
$$


Esta ecuación describe el comportamiento de la solidificación de la aleación, con perdida de calor por conducción y convexión.

\section{$\underline{\text { Curva de enfriamiento }}$}

A partir del balance de calor, podemos hallar la velocidad de enfriamiento:

$$
\begin{aligned}
& \frac{d Q_{L}}{d t}-V \rho C_{p} \frac{d T}{d t}=h A_{S}\left(T-T_{a m b} .\right) \\
& \frac{d T}{d t}=\frac{1}{V \rho C_{p}}\left(\frac{d Q_{L}}{d t}-h A_{S}\left(T-T_{a m b} .\right)\right)
\end{aligned}
$$

Si no ocurre cambio de fase, entones se obtiene una expresión que se denomina curva cero:

$$
\frac{d T}{d t}=-\frac{h A_{S}\left(T-T_{a m b}\right)}{V \rho C_{p}}
$$

de la diferencia de las ecuaciones (5) y (6) podemos determinar el calor de transformación de fase durante la solidificación:

$$
Q_{L}=V \rho C_{p} \int_{0}^{t_{s}}\left(\left.\frac{d T}{d t}\right|_{c e}-\left.\frac{d T}{d t}\right|_{c z}\right) d t
$$

en donde:

$$
\text { ce: curva de enfriamiento. }
$$$$
c z \text { : curva cero. }
$$

El calor latente esta relacionado con el calor de transformación, la densidad y volumen del metal, por lo que obtenemos la siguiente expresión:

$$
L=\frac{Q_{L}}{V \rho}=C_{p} \int_{0}^{t_{s}}\left(\left.\frac{d T}{d t}\right|_{c e}-\left.\frac{d T}{d t}\right|_{c z}\right) d t
$$

Por lo tanto el calor latente de solidificación es la integral desde un tiempo $t=0$ hasta el tiempo de solidificación $t_{S}$, de la diferencia de las velocidades de la curva de enfriamiento y la curva cero.

\section{Cantidad de sólido}

La fracción de sólido $F_{S}$ en la solidificación en un tiempo $t$ se determina con la siguiente ecuación:

$$
F_{S}=\frac{\int_{0}^{t}\left(\left.\frac{d T}{d t}\right|_{c e}-\left.\frac{d T}{d t}\right|_{c z}\right) d t}{\int_{0}^{t_{S}}\left(\left.\frac{d T}{d t}\right|_{c e}-\left.\frac{d T}{d t}\right|_{c z}\right) d t}
$$

La expresión representa el área entre velocidades de la curva de enfriamiento y la curva cero en el tiempo $t$ y el área total entre éstas velocidades, evaluadas hasta el tiempo de solidificación.

\subsection{Método Numérico.}

El estudio de la transferencia calórica en un sistema metal-molde puede realizarse mediante soluciones numéricas, consideramos en estas soluciones la transformación de la fase líquida a sólida y la convexión natural entre la fase líquida y pastosa de la aleación. Para poder implementar la solución por medio del método numérico, se procedió a fijar algunas condiciones

- Se simula en forma transiente el proceso de solidificación, incluyendo los efectos del movimiento del fluido.

- Se considera las propiedades del molde constantes y de la aleación variable con la temperatura.

- El modelo empleado solo considera efectos microscópicos que suceden en un proceso real de solidificación, por lo tanto no es capaz de capturar fenómenos como crecimiento dendrítico, crecimiento de núcleos en la zona pastosa y estructura final del material solidificado.

- No se considera el cambio de la concentración que ocurre en una solidificación de una aleación debido a la reyección de soluto que ocurre en el crecimiento de la interfase.

- El flujo del metal se considera en régimen laminar.

- El sistema de ecuaciones se reduce a un sistema bidimensional, ya que existe simetría con el resto de las direcciones. 
En ingeniería, una gran cantidad de fenómenos es gobernada por la ecuación general de difusión en términos de la variable dependiente $T$, el valor del coeficiente de difusión $\Gamma$ y los términos fuente $S$ elegidos. Se utiliza balances de energía (ver ec. (3)) para deducir las condiciones de borde discretizadas. El método consiste en discretizar el sistema aleación-molde en una red de puntos a las cuales se llamaron nodos, a estos nodos se le asocia una solución de la ecuación de la energía que es función de los nodos que lo rodean y del tiempo, el método por el cual se resolverá el sistema discreto de nodos es utilizando el método de volumen finito, para resolver el sistema de ecuaciones generadas por la discretización del metal-molde, se utilizó el método de línea a línea, que consiste en una adecuada combinación del método de TDMA y Gauss-Seidel [6]. La discretización del MVF se muestra en la Fig. 1.

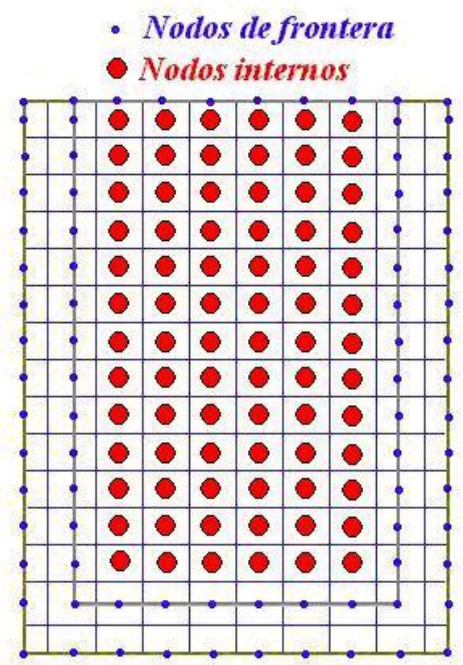

Fig. 1. Gráfica de la Discretización.

Por medio de la función de cambio de fase $f(T)$, se estudia la evolución y liberación progresiva de la entalpía de cambio de fase. Para describir el comportamiento del metal líquido, utilizamos las siguientes ecuaciones:

Ecuación de continuidad

$$
\frac{\partial u}{\partial x}+\frac{\partial v}{\partial z}=0
$$

Ecuación de momento lineal en la dirección $x$ :

$$
\rho\left(\frac{\partial u}{\partial t}+u \frac{\partial u}{\partial x}+v \frac{\partial u}{\partial z}\right)=-\frac{\partial P}{\partial x}+\mu\left(\frac{\partial^{2} u}{\partial x^{2}}+\frac{\partial^{2} u}{\partial z^{2}}\right)
$$

Ecuación de momento lineal en la dirección z:

$$
\rho\left(\frac{\partial v}{\partial t}+u \frac{\partial v}{\partial x}+v \frac{\partial v}{\partial z}\right)=-\frac{\partial P}{\partial z}+\mu\left(\frac{\partial^{2} v}{\partial x^{2}}+\frac{\partial^{2} v}{\partial z^{2}}\right)-\rho g .
$$

Ecuación de difusión de calor:

$$
\begin{aligned}
& \rho C_{p}\left(\frac{\partial T}{\partial t}+u \frac{\partial T}{\partial x}+v \frac{\partial T}{\partial z}\right)= \\
& K\left(\frac{\partial^{2} u}{\partial x^{2}}+\frac{\partial^{2} u}{\partial z^{2}}\right)+\rho L\left(\frac{\partial f_{p c}}{\partial t}+u \frac{\partial f_{p c}}{\partial x}+v \frac{\partial f_{p c}}{\partial z}\right)
\end{aligned} .
$$

Debido a que el último término de la derecha se evalúa sólo en la frontera de la zona pastosa con la zona sólida, las velocidades son casi nulas, dándonos la siguiente ecuación:

$$
\begin{gathered}
\rho C_{p}\left(\frac{\partial T}{\partial t}+u \frac{\partial T}{\partial x}+v \frac{\partial T}{\partial z}\right)= \\
K\left(\frac{\partial^{2} u}{\partial x^{2}}+\frac{\partial^{2} u}{\partial z^{2}}\right)+\rho L \frac{\partial f_{p c}}{\partial t}
\end{gathered}
$$

donde:

$$
f_{p c}=\left\{\begin{array}{ll}
0 & T \leq T_{s} \\
0<g(T) \leq 1 & T_{s}<T \leq T_{l} . \\
1 & T>T_{l}
\end{array} .\right.
$$

La función $g(T)$ representa la forma en que se libera la energía durante el cambio de fase, cuando el metal se encuentra en la llamada zona pastosa el tipo de función cambia de fase elegido para este problema es lineal de la forma:

$$
f_{p c}=\frac{T-T_{s}}{T_{l}-T_{s}}
$$

Para muchos problemas de solidificación de metales se supone que la densidad del metal varía en forma lineal con respecto a la temperatura, por lo que el último término de la ecuación de momento lineal en la dirección $z$ se puede escribir como: 


$$
-\rho g=\rho_{0} g \beta\left(T-T_{r e f}\right)
$$

Por otra parte, las ecuaciones de Navier-Stoke son válidas para la zona líquida y para la zona sólida si se considera viscosidad infinita del metal sólido, el problema se presenta en la zona pastosa. Para solucionar este problema se considerará una estructura equiaxial en el interior de la zona pastosa, ya que existe una aproximación para la viscosidad que producirá el efecto de una zona de transición, así la viscosidad puede escribirse como:

$$
\mu=\frac{\mu_{\text {metal }}}{1-f_{p c}}=\frac{\mu_{\text {metal }}}{f_{l}} .
$$

Entonces cuando el metal esta cerca de la interfase líquido-sólido, el valor de la fracción sólida es cercano a uno, con lo que la viscosidad posee un valor extremadamente alto y la velocidad será cercana a cero, del mismo modo, donde el metal es líquido, la fracción sólida tiende a cero y la velocidad se aproxima a la velocidad del líquido.

El paso de tiempo fue variable, la convergencia se aceleró empleando factores de subrelajación. El criterio de convergencia empleado fue

$$
\left|\frac{\left(T^{k-1}-T^{k}\right)}{T^{k}}\right| \leq \varepsilon
$$

el valor del parámetro de convergencia fue igual a $1 \times 10^{-4}$ para las velocidades y $10^{-2}$ para el cálculo de temperatura.

\section{Experimento}

Para realizar esta experiencia se procedió a fundir la aleación con una composición de $70 \%$ $\mathrm{Pb}$ y $30 \% \mathrm{Sb}$, esta aleación se colocó en una copa de grafito, colocándolo luego en un horno de gas turbulento. La aleación fundida se vació en un molde de arena-resina, cuyas dimensiones son mostradas en la Fig. 2:

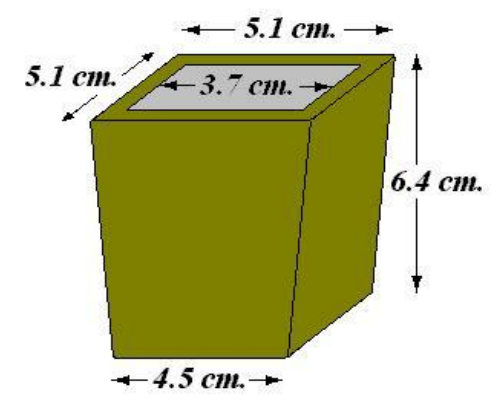

Fig. 2. Molde de Arena y Resina.

Este molde contiene una termocupla a la mitad del interior del molde, esta termocupla es de tipo $K$, para aleaciones no ferrosas, éste está conectado a una interfase y éste a un computador, que registra los datos administrados por la interfase que mide la velocidad de enfriamiento, es decir, la temperatura de enfriamiento y el tiempo transcurrido, se procedió a vaciar en tres diferentes temperaturas de colada siendo estas de $475^{\circ} \mathrm{C}, 431^{\circ} \mathrm{C}$ y $377^{\circ} \mathrm{C}$.

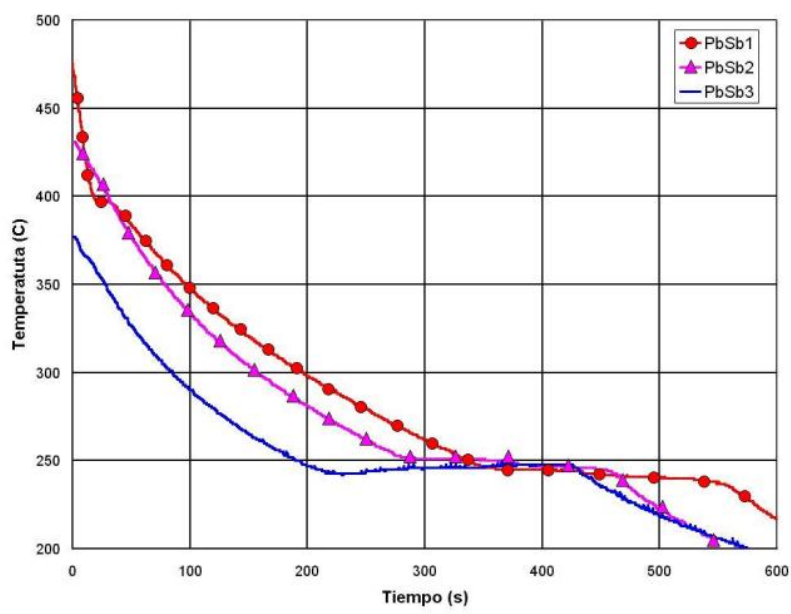

Fig. 3. Curvas de enfriamiento $\mathrm{PbSb}$

Una vez enfriado los 3 moldes con la aleación, se procedió a sacar un trozo de la parte interior de la muestra solidificada, cortándose con una sierra. Las muestras fueron pulidas con diferentes lijas, de grano grueso a fino, el pulido fue hecho con un disco de pulido marca Buehler, no fue necesario atacar químicamente a las muestras, pudiéndose observar la microestructura a simple vista, se tomaron tres fotos en diferentes aumentos, con un equipo de espectrometría de emisión óptica marca Spectro. Para el cálculo 
analítico del calor latente de fusión, se utilizó la expresión (6), si asumimos que las propiedades de la aleación y de la convexión del aire son constantes, entonces:

$$
K=\frac{h A_{s}}{V \rho C_{p}}
$$

obteniéndose que:

$$
\frac{d T}{d t}=-K\left(T-T_{a m b}\right)
$$

integrando tenemos la siguiente expresión:

$$
T=T_{a m b}+\left(T_{c}-T_{a m b},\right) e^{-K t}
$$

donde:

$\begin{array}{ll}T_{c}: & \text { temperatura de colada. } \\ T_{a m b .}: & \text { temperatura ambiente. }\end{array}$

Para obtener los datos para el ajuste de la curva cero, se seleccionó aleatoriamente 3 puntos de la línea líquidus de la curva de enfriamiento experimental, como podemos ver en la Tabla 1:

Tabla 1. Datos de 3 puntos aleatorios

\begin{tabular}{ccc}
\hline Muestra & $\boldsymbol{T}\left({ }^{\circ} \boldsymbol{C}\right)$ & $\boldsymbol{T}(\boldsymbol{s})$ \\
\hline & 385 & 50 \\
PbSb(1) & 349 & 100 \\
& 321 & 150 \\
& 412 & 20 \\
$\operatorname{PbSb}(2)$ & 377 & 50 \\
& 304 & 150 \\
& 347 & 30 \\
$\operatorname{PbSb}(3)$ & 327 & 50 \\
& 265 & 150 \\
\hline
\end{tabular}

Para el cálculo del calor latente se utilizo el dato de $C_{p}=5.594$ ( $\left.\mathrm{cal} / \mathrm{mol}-\mathrm{K}\right)$, de la aleación $P b S b$.Para la simulación del comportamiento de enfriamiento de la aleación en el molde, se utilizaron las siguientes condiciones iniciales y de frontera:

\section{Condiciones iniciales:}

\section{Aleación:}

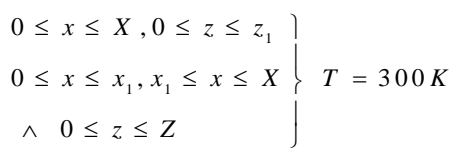

molde:

$$
\left.x_{1} \leq x \leq x_{2} \wedge y_{1} 0 \leq y \leq Y\right\} T=T_{c}
$$

\section{Condiciones de frontera:}

Interfase molde-ambiente:

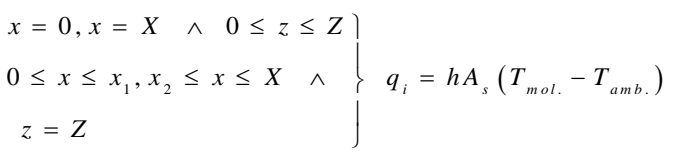

interfase aleación-molde:

$$
\left.\begin{array}{l}
x_{1} \leq x \leq x_{2} \wedge z=z_{1} \\
x=x_{1}, x=x_{2} \wedge z_{1} \leq z \leq Z
\end{array}\right\} \quad q_{i}=-K A \frac{\partial T}{\partial t}
$$

interfase aleación-ambiente:

$\left.x_{1} \leq x \leq x_{2} \wedge z=Z\right\} \quad q_{i}=h A_{s}\left(T_{a l .}-T_{a m b .}\right)$

Tabla 3: Datos para la modelación numérica

\begin{tabular}{lc}
\hline Aleación PbSb & \\
\hline Cond. térmica $(\mathrm{W} / \mathrm{mK})$ & 27.3 \\
Calor esp. $\mathrm{Cp}(\mathrm{J} / \mathrm{Kg} \mathrm{K})$ & $5.594+.12 * 10^{-3} \mathrm{~T}$ \\
Densidad $\rho\left(\mathrm{Kg} / \mathrm{m}^{3}\right)$ & 9946.7 \\
Visc. dinámica $(\mathrm{Kg} / \mathrm{ms})$ & 0.045 \\
\hline Molde de arena-resina & 1 \\
\hline Conductividad térmica $\mathrm{K}(\mathrm{W} / \mathrm{mK})$ & 1300 \\
Calor específico $\mathrm{Cp}(\mathrm{J} / \mathrm{KgK})$ & 2000 \\
\hline Densidad $\rho\left(\mathrm{Kg} / \mathrm{m}^{3}\right)$ &
\end{tabular}

\section{Resultados}

En esta sección se presentan los diferentes resultados obtenidos en el análisis numérico y metalográfico del sistema $\mathrm{PbSb}$.

\subsection{Resultados analíticos}

\begin{tabular}{|c|c|c|c|c|c|}
\hline Muestra & $T\left({ }^{\circ} \mathrm{C}\right)$ & $t(s)$ & $K^{*} 10^{3}$ & $K_{c o} * 10^{3}$ & $T_{c}\left({ }^{\circ} \mathrm{C}\right)$ \\
\hline \multirow{3}{*}{$\operatorname{PbSb}(1)$} & 385 & 50 & 7.93 & \multirow{3}{*}{5.47} & \multirow{3}{*}{475} \\
\hline & 349 & 100 & 6.13 & & \\
\hline & 321 & 150 & 5.47 & & \\
\hline \multirow{3}{*}{$\operatorname{PbSb}(2)$} & 412 & 20 & 4.29 & \multirow{3}{*}{5.32} & \multirow{3}{*}{431} \\
\hline & 377 & 50 & 5.33 & & \\
\hline & 304 & 150 & 5.32 & & \\
\hline \multirow{3}{*}{$\operatorname{PbSb}(3)$} & 347 & 30 & 6.19 & \multirow{3}{*}{6.68} & \multirow{3}{*}{377} \\
\hline & 327 & 50 & 6.64 & & \\
\hline & 265 & 150 & 6.68 & & \\
\hline
\end{tabular}

De la curva de enfriamiento, obtenido durante el experimento y utilizando la ecuación (20), obtenemos el valor de $K$, que identifica las propiedades de la aleación:

Tabla 4: Propiedades de la Aleación

El área entre las curvas se calculó entre las diferencias de las integrales de las velocidades de 
la curva de enfriamiento y de la curva cero, ecuación (8), obteniéndose el calor latente de solidificación:

Tabla 5: Calor Latente de la Aleación:

\begin{tabular}{ccccc}
\hline Muestra & $\boldsymbol{A}_{c e}$ & $\boldsymbol{A}_{c o}$ & $\boldsymbol{\Delta A}$ & $\boldsymbol{L}_{f}(\mathbf{c a l} / \mathbf{m o l})$ \\
\hline $\operatorname{PbSb}(1)$ & 258.06 & 264.56 & 6.54 & 36.58 \\
$\operatorname{PbSb}(2)$ & 226.44 & 221.43 & 5.01 & 28.02 \\
$\operatorname{PbSb(3)}$ & 178.66 & 173.69 & 5.01 & 28.02 \\
\hline
\end{tabular}

\subsection{Resultado numérico}

Se presentan los resultados obtenidos en el problema de solidificación no isotérmica de la aleación $P b S b$ en un molde de arena-resina, para una temperatura superior al experimental. Los siguientes gráficos que se presentan son los diferentes perfiles de temperatura, isotermas y vectores de velocidad para diversos tiempos. Perfiles de temperatura, isotermas de la aleaciónmolde y vectores de velocidad por convexión natural en los tiempos de 2, 35 y 100 segundos en la aleación $P b S b$.

\subsection{Resultado del análisis metalográfico.}

Las siguientes fotos fueron tomadas en el laboratorio de metalografía (con el espectrómetro de emisión óptica), luego de ser lijadas y pulidas en el disco Buehler. Las tomas fotográficas realizadas a las tres muestras con diferentes temperaturas de colada de la aleación $\mathrm{PbSb}$, corresponden a los diferentes aumentos utilizados (50X, 100X, 200X) durante el análisis metalográficos. e realizó un análisis a través del diagrama de fase, para relacionarla con la microestructura obtenida en el experimento. En la siguiente Fig. se presenta el diagrama de fase y la microestructura de la aleación $P b S b$ : Podemos suponer que esto es una expresión del equilibrio dado a una velocidad de enfriamiento menor. La primera derivada de la curva de enfriamiento tiene valores irregulares por lo que se tuvo que ajustar, sin embargo las fluctuaciones siguieron presentes, a pesar de esto, se pudo hacer una aproximación al calculo del calor latente de la aleación. El calor latente obtenido por calculo no es la correcto, ya que es mucho menor que el calor de $\mathrm{Pb}$ y Sb, este resultado esta directamente relacionado al sistema fuera del equilibrio. $\mathrm{Si}$ observamos bien los aumentos 200X, vemos que existe una mayor cantidad de dendrítas en la muestra 1 que en la 2 y que en la 3 . Esto es debido a que la velocidad de enfriamiento en la muestra 1 es mas alta, por lo que generará mayor cantidad de núcleos, otro factor que ayuda a la formación de núcleos es la convexión natural que existe en el líquido. Este conjunto de dendrítas es la solidificación de $\beta$ eutéctico, que se encuentra rodeado de $\alpha$, que en conjunto forma la estructura eutéctica y los granos forman el $\beta$ primario. A fin de modelar y explicar la dinámica seguida por el enfriamiento mediante algún tipo de información, debería poder controlar y medir un importante conjunto de situaciones o condiciones experimentales, por ejemplo, la temperatura de la atmósfera, la linealidad del instrumento de medición empleado, el rango de temperatura medida, el proceso matemático digital de la señal y la inducción de errores en el cableado. Durante el enfriamiento, la disipación de calor en la muestra 1 es por conducción, cuando se llega a esta temperatura de seudo equilibrio, la disipación de calor es ahora por convexión del medio que lo rodea, mientras que en la muestra 3 , la aleación y el molde no llegan al seudo equilibrio térmico, por lo que la disipación es fuertemente por convexión. Para los primeros tiempos de iniciado el proceso de solidificación el núcleo de metal fundido se desplaza hacia la parte superior de la cavidad debido al efecto de la convección natural, transcurridos más de $100 \mathrm{~s}$ se observa la forma curva de las isotermas ubicadas en la parte inferior de la cavidad, esto se debe a que la parte inferior del molde se encuentra aislada. La formación de dos vórtices simétricos y alargados encontrados durante el proceso de solidificación reflejan la influencia de la mecánica de fluidos durante el proceso, sin embargo no se encuentra un desplazamiento notorio junto con el núcleo caliente de metal hacia la parte superior del molde. 


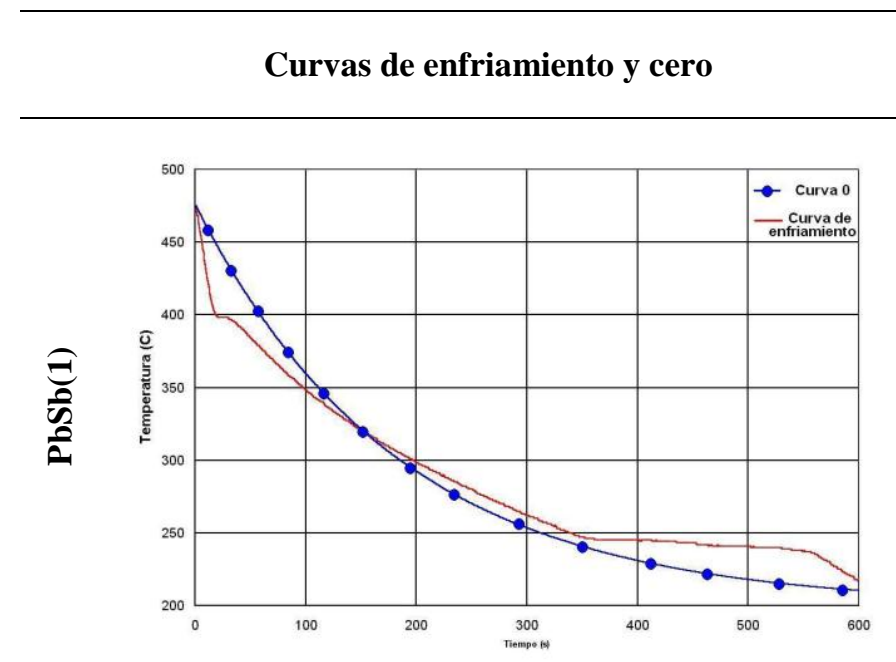

\section{Velocidades de enfriamiento}
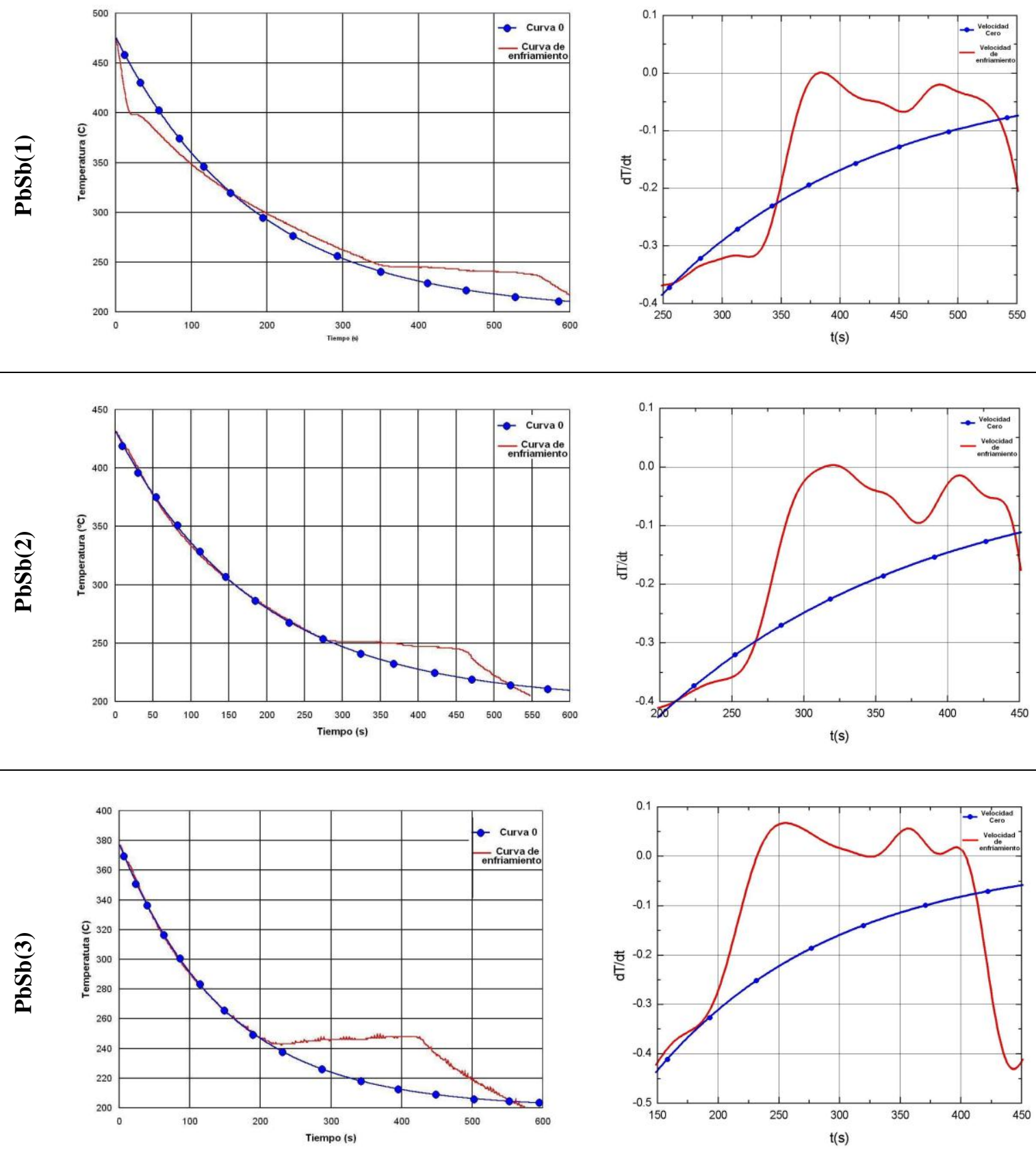

Fig. 4. Cálculo de la curva cero a partir de la ecuación (22), la derivada de la curva de enfriamiento y la curva cero 


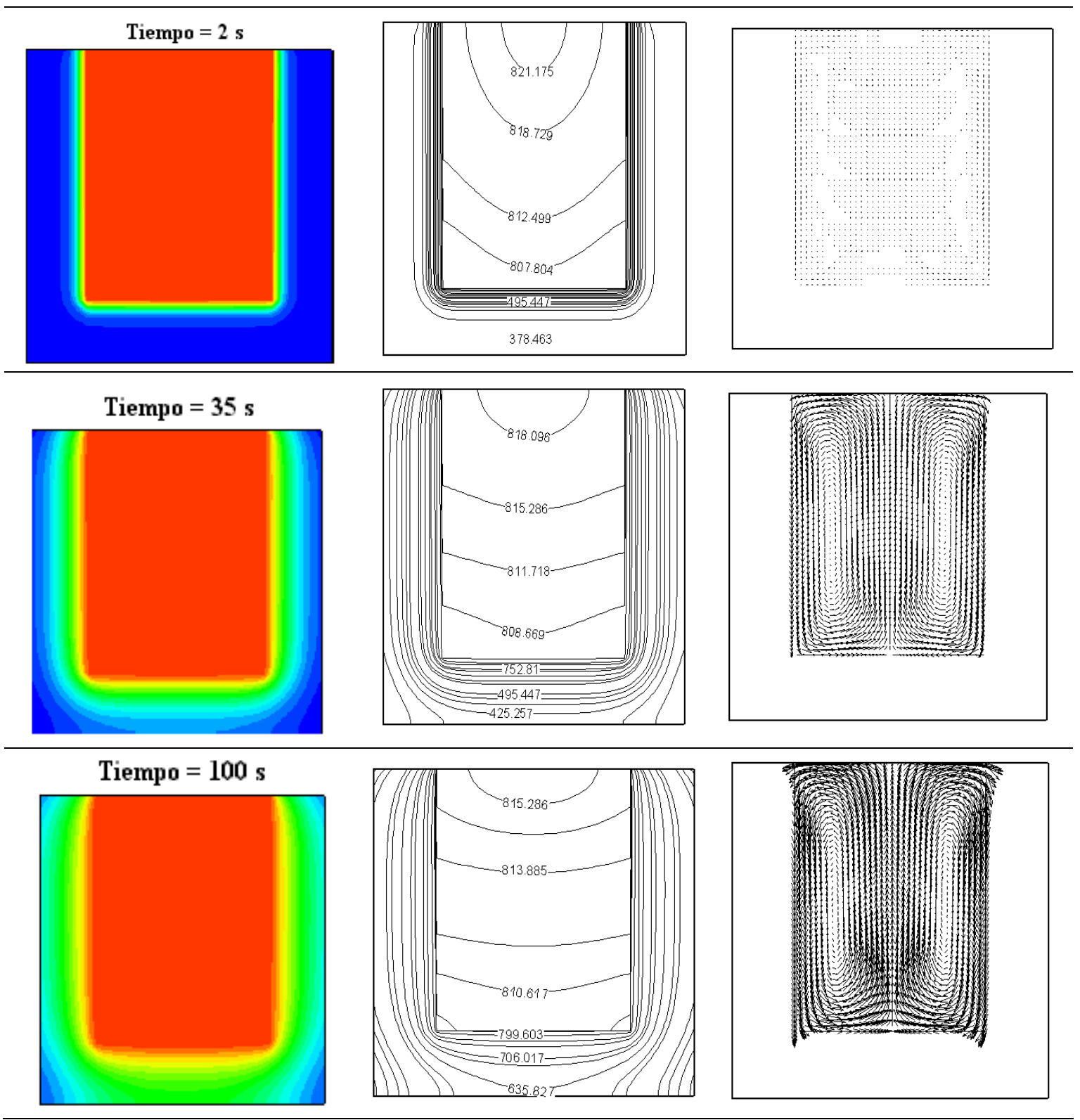

Fig. 5. Gráficos de los diferentes perfiles de temperatura, isotermas y vectores de velocidad para tiempos de $2 \mathrm{~s}, 35 \mathrm{~s} \mathrm{y} 100 \mathrm{~s}$. 


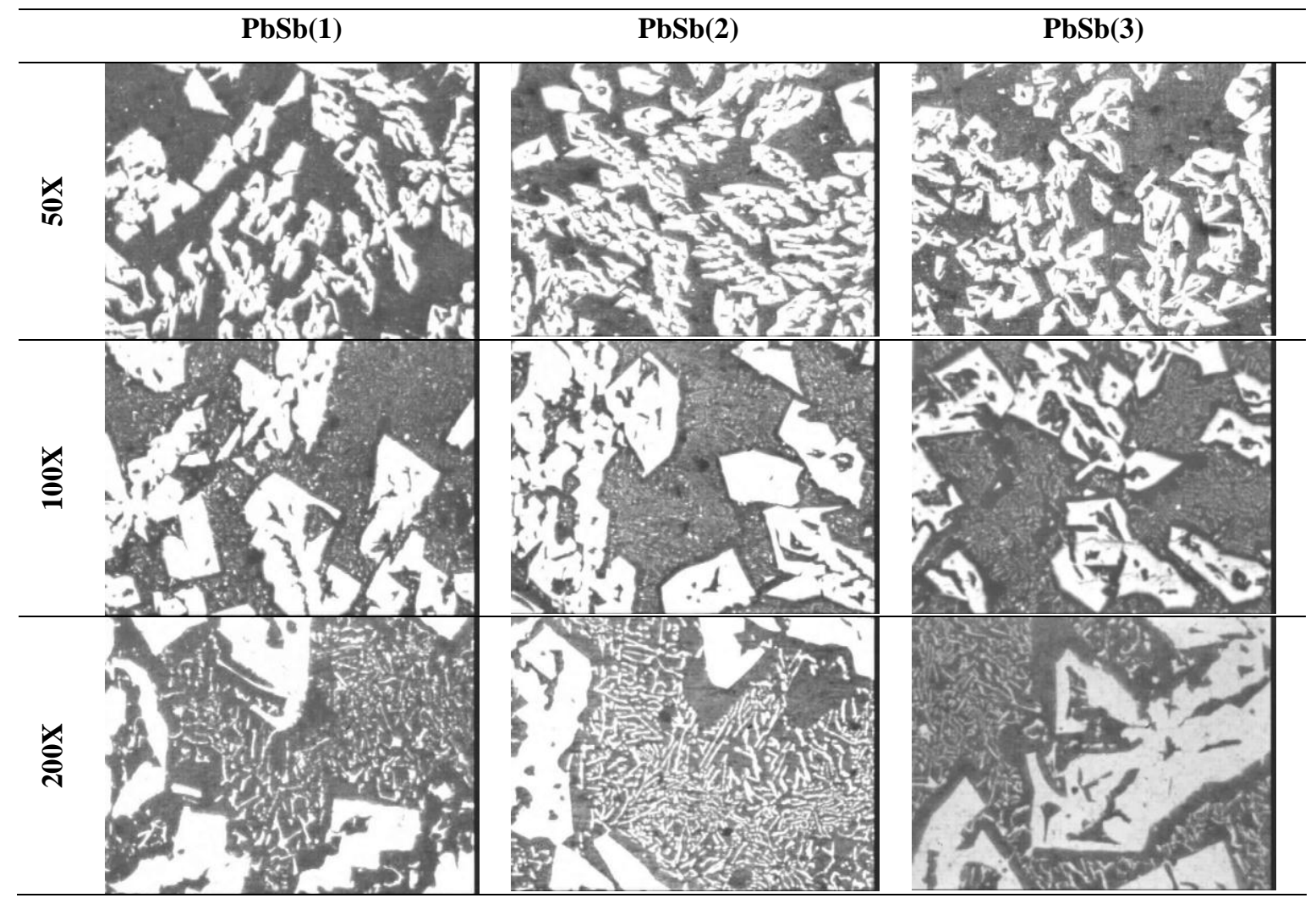

Fig. 6. Fotografías de las tres muestras con diferentes temperaturas de colada de la aleación $P b S b$, correspondientes a los 3 aumentos (50X, 100X, 200X).

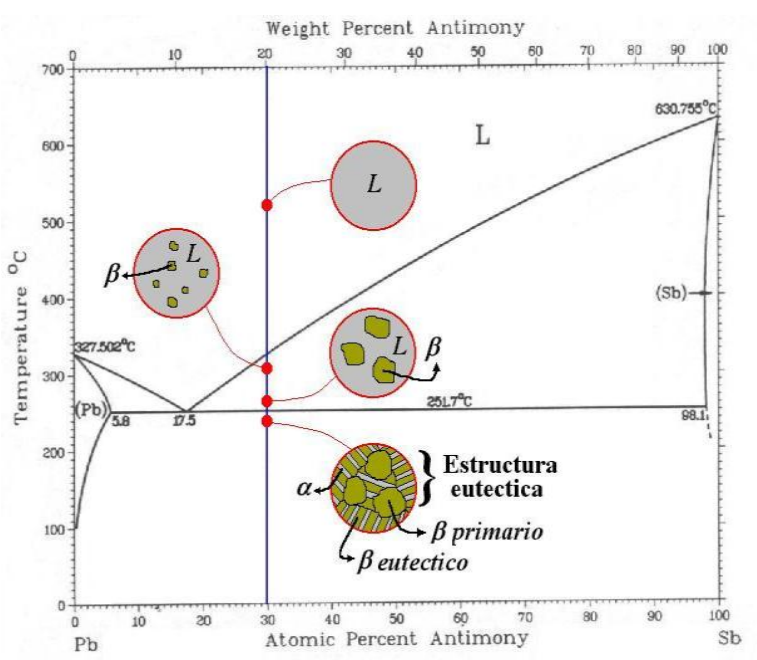

Fig. 7. Diagrama de fase y la microestructura de la aleación $P b S b$

\section{Conclusión}

$\mathrm{Al}$ reunir las tres curvas de enfriamiento observamos que estos están a diferentes temperaturas de colada, por lo que tienen velocidades de enfriamiento diferentes, lo que quiere decir que la velocidad de enfriamiento en la muestra 1 será más rápida, pero sin embargo, necesitara mayor tiempo para poder solidificar, debido que la velocidad decrece exponencialmente con respecto al tiempo.

La curva de solidificación obtenida es una curva de equilibrio dinámico, su análisis entrega parámetros importantes del proceso de solidificación, como calor latente, crecimiento 
granular en la zona pastosa $(L+\beta)$ y calor de transformación de fase.

Del diagrama de fase en equilibrio, observamos que la zona pastosa para este proceso de noequilibrio, comienza a temperaturas mucho menores que lo esperado y el rango de temperatura es mucho mas estrecho.

Cuando el líquido cruza la linea líquidus del diagrama de fase $\mathrm{PbSb}$, la fase $\beta$ empieza a precipitar, debido a que hay una saturación de ésta, formándose la fase $\beta$ primaria, conforme la aleación sigue enfriándose, la fase $\beta$ sigue creciendo formando un grano mas grande, rodeado de líquido, al pasar la temperatura eutéctica, todo el liquido se ha solidificado, formándose 2 fases sólidas, característica de los hipereutecticos, el grano de la fase $\beta$ dejo de crecer y alrededor se encuentra una estructura que se compone de $\beta$ eutéctico y la fase $\alpha$, esta nueva estructura se conoce como estructura eutéctica.

La teoría nos indica que la microestructura de un lingote tiene 3 zonas definidas, una en la vecindad de la pared del molde, en donde el grano es nodular pequeña, una zona media en donde se da el crecimiento dendrítico, una zona en donde el grano es nodular equiaxial, que se explica por nucleación rápida, direccionalidad de la transferencia de calor en las dendrítas y reyección de soluto a la interfase sólido-líquido.

- Al analizar las fotos $100 x$, observamos que el tamaño de grano del amonio es un poco mas grande en la muestra 3 que en la 2 y 1 , y que el grano en la 2 es mas grande que en la 1 . el enfriamiento de las muestras, la velocidad será mayor cuando las diferencias de temperatura son muy grandes, con las mismas condiciones de frontera.

- Se observaron tamaños de granos mayores a medida que la temperatura de colada es menor. El tamaño de grano es más grande conforme la velocidad de enfriamiento es más lento.
- La cantidad de dendrítas esta directamente relacionada a la velocidad de enfriamiento.

- El calor latente obtenido se pudo calcular solo con la diferencia de la integral de las áreas de las velocidades de enfriamiento, de la curva cero y con el calor específico de la aleación.

- La estructura hipereutectica genera 2 fases sólidas, en la que existe una estructura eutéctica, formado por las fases $\alpha$ y $\beta$ eutéctico, y la fase $\beta$ primaria.

- Las zonas claras en las fotos, es la fase $\beta$ primaria, mientras que la zona oscura es la fase $\alpha$.

\section{AGRADECIMIENTOS}

Agradecemos a la Unidad de Postgrado de la Facultad de Ingeniería de la Universidad Santiago de Chile por su apoyo en la fase experimental. Especial agradecimiento a la Unidad de Post Grado de la Facultad de Ciencias Físicas de la UNMSM por su apoyo con el Laboratorio de Cálculo Científico.

\section{Referencias}

[1]. D. R. Askeland, La Ciencia e Ingeniería de los Materiales, Grupo Editorial Ibero América, (México 1987).

[2]. Richard Flinn, Fundamentals of Metal Casting, (1967).

[3]. Bruce Chalmers, Principles of Solidification, (1972.)

[4]. Robert. E. Hill, Principios de Metalurgia Fisica, CECSA. (Mexico 1967).

[5]. Avner Sydney, Introducción a la Metalurgia Física, McGraw-Hill, (México).

[6]. Guillermo Sánchez M.1 César Vial R.2 Nelson Moraga B. Estudio de Fluidos no Newtonianos con los Métodos de Volúmenes y Elementos Finitos. Revista Facultad de Ingeniería, U.T.A. (CHILE), VOL 10, 2002, pp. 23-34. 